Torreón Universitario

www.faremcarazo.unan.edu.ni / ISSN 2410-5708 / e-ISSN 2313-7215

Año 9 | Núm. 24 | pág. 16 - pág. 26 | febrero - mayo 2020
Esta obra está bajo una licencia de Creative Commons Atribución-NoComercial-SinDerivar 4.0 Internacional

\title{
Actividad biocida de extractos de guanábana (Annona muricata L.) en el estadio larval III del mosquito Aedes aegypti L.
}

\author{
Lic. Nohemí Carballo Castillo \\ Tesista \\ Laboratorio de Biotecnología, UNAN-Managua \\ ecnohemicastillo1@gmail.com
}

\section{Lic. Diana Bermúdez Hernández}

Tesista

Laboratorio de Biotecnología, UNAN-Managua

dianaes94h@gmail.com

\section{M.Sc. Samantha Miranda Calero \\ Investigadora \\ Laboratorio de Biotecnología, UNAN-Managua \\ smiranda@unan.edu.ni}

\section{M.Sc. Ena Rivers Carcache}

Investigadora

Laboratorio de Biotecnología, UNAN-Managua erivers@unan.edu.ni

\section{Ph.D. Martha Lacayo Romero}

Investigadora

Laboratorio de Biotecnología, UNAN-Managua biotecnologia@unan.edu.ni

Ph.D. Lester Rocha

Docente investigador

Facultad de Ciencia Animal, UNA

lester.rocha@ci.una.edu.ni

Fecha de recepción: 13 de febrero, 2020 / Fecha de aceptación: 24 de febrero, 2020

https://doi.org/10.5377/torreon.v9i24.9721

Palabras clave: Guanábana, Annona muricata L., Aedes aegypti L., larvicida, semilla.

\section{RESUMEN}

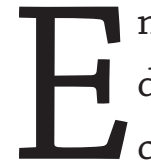

n la actualidad el mosquito (Aedes aegypti) es el principal vector para la transmisión de virus causantes de enfermedades infecciosas de alta reactividad patogénica como el dengue, chikungunya y zika, que asociadas a factores de riesgo o poca atención médica pueden ser letales. Adicionalmente los sistemas de salud se enfrentan con el creciente problema de la carencia de un método de control de este mosquito que prolifera fácilmente y que cada vez presenta mayor resistencia a insecticidas históricamente efectivos, 
limitando las opciones disponibles para su erradicación, constituyendo un punto clave en la salud pública mundial. La presente investigación evaluó la actividad larvicida de extractos de hoja y semilla de guanábana (Annona muricata L.) obtenido mediante dos métodos de extracción (Soxhlet y reposo) como una alternativa para el control del A. aegypti en su estadio larvario III. De igual manera se evalúo el efecto del solvente utilizado para la obtención de los extractos y el efecto de las condiciones del medio y ambientales sobre la mortalidad de la larva. Los resultados reflejaron que las diferencias existentes en la composición proximal de las muestras dependen no solo de la matriz (hoja o semilla) sino también de la procedencia de las mismas. Asimismo, se constata el efecto larvicida de los extractos de semilla y hoja de guanábana sobre el estadio larval III del mosquito, sin embargo su efectividad está en dependencia del método de extracción. Los controles indicaron que el ambiente y el medio no influyeron en el momento de evaluar la letalidad.

\section{INTRODUCCIÓN}

En Nicaragua a partir del año 2013 se ha registrado un incremento en las tasas de personas afectadas por el mosquito Aedes aegypti L. constituyendo uno de los principales problemas de salud pública en el país debido a las arbovirosis transmitidas por este mosquito, tales como el dengue, zika, chikungunya y fiebre amarilla (Alfaro J., 2017).

La Organización Mundial de la Salud (OMS) ha normado diversas medidas para el control de este vector, entre las cuales se encuentra el uso de químicos aplicados en las distintas fases de su ciclo, entre ellos temefos (Abate ${ }^{\circledR}$ ) en estadio larval y cipermetrina en estado adulto mediante una técnica especializada nombrada rociado especial.

Actualmente el principal problema con el uso y la práctica excesiva de estos compuestos es el incremento en la contaminación ambiental además de la creciente resistencia de este organismo al uso de Abate $^{\circledR}$, permitiendo que el mosquito logre desarrollar todo su ciclo (Castillo J. y Ramos J., 2016).

Por lo antes mencionado resulta necesaria la evaluación de nuevas opciones para el control del vector que generen un menor impacto ambiental, representen un menor riesgo a la salud humana y disminuyan la generación de resistencia. Dentro de estas potenciales nuevas alternativas se encuentra a la guanábana (Annona muricata L). Esta especie de planta posee importancia alimenticia, medicinal e industrial asociada a los compuestos activos presentes en todas las partes de la planta entre los cuales destacan las acetogeninas, metabolitos a los cuales se atribuyen sus propiedades bioinsecticidas y plaguicidas.

El presente estudio realizó la caracterización proximal de las muestras utilizadas para la obtención de extractos y evalúo el potencial de la hoja y semilla de la guanábana nacional a 
escala de laboratorio para el control del tercer estadio larval del $A$. aegypti con el propósito de determinar dosis efectivas que el organismo en estudio no tolere desde el ciclo inmaduro. Este proceso se realizó estableciendo pruebas de toxicidad (bioensayos) para determinar el potencial biocida de los extractos obtenidos mediante los métodos de extracción: reposo y Soxhlet.

\section{MATERIALES Y MÉTODOS}

\section{Colecta de las muestras}

Se colectaron 20 frutos de guanábana en el comercio de Managua (Mercado Oriental y Mercado Mayoreo). Las muestras fueron desinfectadas, transportadas y procesadas en el Laboratorio de Biotecnología de la UNAN-Managua en condiciones controladas para evitar su degradación. Las semillas fueron extraídas del fruto, se lavaron con agua potable y agua destilada, se secaron a temperatura ambiente durante 7 días y en horno de convección a $40^{\circ} \mathrm{C}$ durante 24 horas, posteriormente se homogenizó la muestra en un molino RETSCH con tamaño de poro de $0.5 \mathrm{~mm}$. La harina obtenida se almacenó en recipientes herméticos color ámbar a $4^{\circ} \mathrm{C}$.

Las hojas utilizadas en el estudio fueron obtenidas de 5 árboles del Centro de Desarrollo Tecnológico (CDT) Campos Azules tomando en cuenta sus condiciones fitosanitarias. De cada árbol se seleccionó aleatoriamente una cantidad representativa de hojas (aproximadamente 100) tomando en cuenta su edad y calidad (libre de plagas o enfermedades).

\section{Caracterización morfológica de las muestras}

Los frutos, semillas y hojas fueron caracterizados utilizando los marcadores morfológicos establecidos por Bioversity International y CHERLA (2008) para guanábana. Algunos de los parámetros para fruta, hoja y semilla fueron: longitud, diámetro, peso del fruto, peso de semillas, así como características anatómicas cáscara (exocarpio), pulpa (mesocarpio).

\section{Determinación del contenido proximal de la semilla}

La composición química se obtuvo utilizando metodologías establecidas por la AOAC. La determinación de humedad, cenizas y extracto etéreo se realizaron según: AOAC 925.1, AOAC 923.03 y AOAC 920.39, respectivamente. Todos los análisis se realizaron por triplicado con controles negativos (blancos) y controles de calidad internos. Los datos obtenidos fueron evaluados para determinar su reproducibilidad y exactitud.

\section{Preparación de extractos}

Posterior a la caracterización del fruto se extrajeron sus semillas y su transformaron en harina y se homogenizó la muestra de la cual se utilizaron 25 g para cada extracto. De igual manera las hojas se transformaron en harinas y del peso final homogenizado se utilizaron $25 \mathrm{~g}$ para cada extracto producido. 
Los extractos evaluados fueron preparados por los métodos de reposo y Soxhlet en triplicado. En el método de reposo la harina se colocó en frascos de vidrios ámbar con el solvente (etanol 96\%) y permaneció por 7 días a temperatura ambiente. Posteriormente fue filtrado hasta obtener la solución y se almacenó en frascos ámbar a $4^{\circ} \mathrm{C}$.

Para el método Soxhlet la harina se colocó en un dedal de hemicelulosa dentro del equipo Soxhlet y se extrajeron con el solvente (etanol 96\%) en períodos distintos de tiempo debido a la diferencia en la naturaleza oleosa de las matrices. En la matriz hoja el tiempo de extracción fue de 8 horas, mientras que en semillas la extracción demoró 16 horas. Posteriormente los extractos obtenidos se almacenaron en frascos oscuros a $4^{\circ} \mathrm{C}$.

\section{Pie de cría del espécimen en estudio}

Para garantizar la identidad del mosquito, los huevos de larvas se obtuvieron en el Complejo Nacional de Salud Concepción Palacios donde se realiza la selección de los mismos de acuerdo a sus características morfológicas. Cinco masas de huevos de 100 individuos aproximadamente (totalizando 500 individuos) fueron eclosionados para el proceso de reproducción en el Laboratorio de Biotecnología de la UNAN-Managua en condiciones controladas (temperatura de $27^{\circ} \mathrm{C}$ a $30^{\circ} \mathrm{C}$ y una de humedad relativa de $70 \%$ ).

Desde el primer estadio se les proporcionó alimento y se reemplazó cada tres días, al igual que el agua debido a los desechos expuestos (mudas). Terminada la fase larval pasan a estado pupa manteniéndose sin alimento por 2 días, durante esta fase fueron resguardados en una cámara entomológica, esperando la eclosión de los mosquitos adultos que fueron utilizados como progenitores para la creación del pie de cría necesario para la realización de los bioensayos.

\section{Diseño y condiciones de los bioensayos}

Fueron diseñados dos bioensayos. En el primer bioensayo, se utilizó un diseño completamente al azar con arreglo factorial de tratamientos, donde los factores evaluados fueron matriz (extractos de hojas y semillas), tipos de métodos de extracción (reposo y Soxhlet) y concentración de extractos de A. muricata (100, 750, 150, 3000, 5000 ppm). Para cada combinación de tratamientos se usaron cinco recipientes como repeticiones que contenían 10 individuos de A. aegypti en III estadio larval. Las lecturas de mortalidad se registraron cada 8 horas (08h, 16h, 24h, 32h, 40h y 4h) posterior a la exposición de los tratamientos. Los individuos fueron alimentados una sola vez durante el período de evaluación.

Paralelamente, en el segundo bioensayo se utilizó un diseño completamente al azar para comprobar el efecto de controles: control negativo (agua), control positivo (Abate ${ }^{\circledR}$ ) y control reactivo (etanol 96\%), sobre la mortalidad de individuos. Tanto el número de repeticiones (y 
número de individuos por recipiente) como los tiempos de las mediciones fueron iguales que aquellos usados en el bioensayo.

Se monitoreó continuamente la temperatura y el $\mathrm{pH}$ de los medios, así como la temperatura y la humedad relativa del ambiente para conocer la relación entre los parámetros: humedad relativa y temperatura ambiental, así como el $\mathrm{pH}$ y la conductividad de los medios con la finalidad de conocer su efecto sobre los resultados obtenidos.

\section{Procesamiento y análisis de los datos}

Para conocer el efecto de la concentración de extractos, del método de extracción y la matriz en el porcentaje de mortalidad de individuos de A. aegypti se utilizó un modelo lineal mixto generalizado con una función de error Binomial. Los factores anteriores fueron considerados factores de efectos fijos, y los factores tiempo de medición y repeticiones como factores de efectos aleatorios.

El modelo completo consistió en el ajuste de efectos principales e interacciones. En todos los casos, después de ajustar el modelo completo, los términos no significativos fueron removidos paso a paso y los sub-modelos fueron comparados usando la prueba de razón de verosimilitud y el criterio de información de Akaike (AIC) como medida para comparar el ajuste de los diferentes modelos. El modelo con AIC más bajo se consideró ser el modelo más parsimonioso. Debido a la presencia de sobre dispersión en el modelo ajustado, se incluyó una variable sintética (modelada como factor de efecto aleatorio) que capturara la sobre-dispersión presente.

Para comprobar el efecto de los controles sobre el porcentaje de mortalidad de individuos de A. aegypti se utlizó un modelo lineal mixto generalizado con una función de error Binomial. El factor solvente de extracción fue considerado efecto fijo y los factores tiempo de medición y repeticiones fueron considerados factores de efectos aleatorios. Debido a la presencia de sobredispersión en el modelo ajustado, se incluyó una variable sintética (modelada como factor de efecto aleatorio) que capturara la sobre-dispersión presente. Fueron realizadas comparaciones múltiples usando la prueba honesta de Tukey. Todos los análisis fueron realizados con el software estadístico R (R Core Team, 2019).

\section{RESULTADOS Y DISCUSIÓN}

\section{Composición proximal}

La materia seca de la hoja fue $90.46( \pm 0.10 \%)$ y en semilla de $96.16( \pm 0.07 \%)$, por tanto el contenido de humedad determinado fue variante para ambas matrices $(9.54 \pm 0.10 \%$ en hoja y $3.84 \pm 0.07 \%$ en semillas). Esto es de gran utilidad para determinar la estabilidad de las matrices en estudio. Por otro lado, el contenido de cenizas presentó un valor promedio en hojas 
de $8.38 \pm 0.13 \%$ y $1.43 \pm 0.006 \%$ en semillas. En cuanto al extracto etéreo, la semilla presentó un mayor contenido $(32.46 \pm 0.06 \%)$ con respecto a la hoja (3.86 $\pm 0.01 \%)$ (Figura 1).

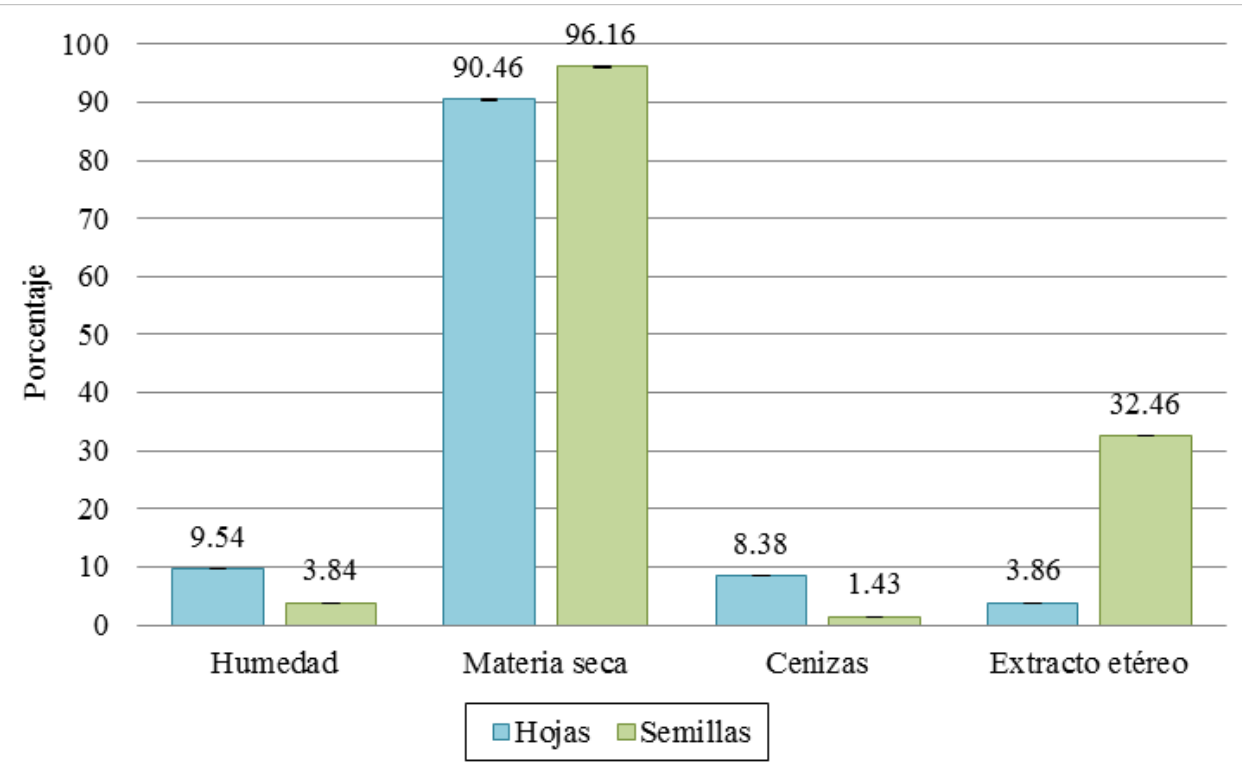

Figura 1. Contenido de humedad, materia seca, cenizas y extracto etéreo de las muestras de A. muricata.

Al contrastar los resultados obtenidos en la composición proximal de la hoja con respecto a lo establecido por Cuello et al. (2017) se encontró similitud en el contenido de cenizas (8.71\%) mientras que existe divergencia en el extracto etéreo $(8.48 \%)$ y la humedad (2.74\%) establecida en esta referencia.

Por otro lado, en la comparación de referencias internacionales de la composición de la semilla de guanábana, encontramos que los valores obtenidos de cenizas presentan gran similitud a los descrito por Vit et al. (2014) en Venezuela (1.44\%) y diferencia a lo establecido por Fasakin et al. (2007) en Nigeria (2.29\%). El contenido de extracto etéreo determinado por Vit et al. (2014) (25.75\%) es similar al descrito por Fasaki et al. (2007) (22.57\%), sin embargo difieren con respecto a los resultados obtenidos en este estudio (32.46\%). La semilla nacional también reporta mayor contenido de materia seca que el obtenido por Vit et al. (2014) (90.13\%).

La variación en el contenido proximal de las matrices se encuentra asociado a las condiciones edafoclimáticas en que la planta se desarrolla. De igual manera la variedad de la guanábana (ácida, sub-ácida o dulce), es un aspecto que a la fecha no ha sido explorado y que puede aportar diferencias significantes en la composición de las muestras en estudio. En la tabla 1 se detalla la clasificación organoléptica de las muestras de fruto que fueron incorporadas en esta investigación. 
Tabla 1. Caracterización organoléptica de las muestras de fruto de guanábana utilizadas.

\begin{tabular}{|l|l|l|l|}
\hline $\begin{array}{l}\text { Cantidad } \\
\text { de frutos }\end{array}$ & Sabor & \multicolumn{1}{|c|}{ Textura } & \multicolumn{1}{c|}{ Color } \\
\hline \multirow{2}{*}{14} & \multirow{2}{*}{ Dulce } & Cremosa & Marrón \\
\cline { 3 - 4 } & & Acuosa & Gris \\
\hline 3 & Ácida & Cremosa & Marrón \\
\hline 3 & Sub ácida & Cremosa & Marrón \\
\hline
\end{tabular}

\section{Evaluación de la letalidad de los extractos etanólicos según el método de extracción y la matriz}

El efecto de la concentración de extractos etanólicos de A. muricata sobre la mortalidad larval varía según el método de extracción $(\mathrm{P}<0.05)$ (Figura 2). Así, las concentraciones de 750 y 1500 ppm obtenidas por el método Soxhlet presentan una mayor letalidad que aquellas obtenidas por el método de reposo ( $\mathrm{P}=0.002$ y $\mathrm{P}=0.004$, respectivamente). Las concentraciones restantes tuvieron un efecto sobre la mortalidad indistintamente del método de extracción. Por otra parte, no se encontró efecto de los extractos de hojas y semillas (matriz) sobre la mortalidad de individuos.

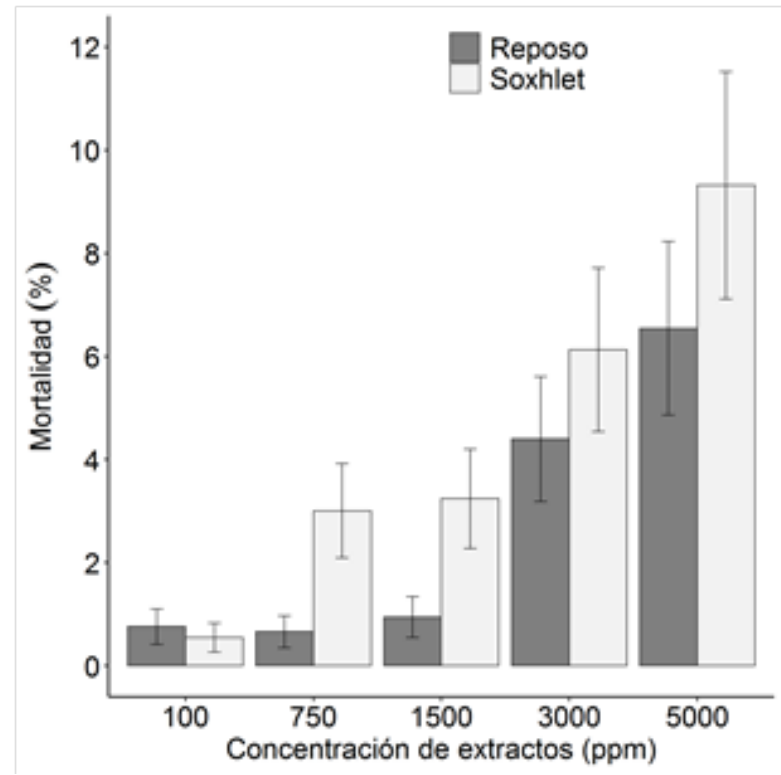

Figura 2. Mortalidad larval media ( \pm 1 error estándar) en función de la concentración de extractos de A. muricata y del método de extracción.

\section{Efecto de los controles en la mortalidad}

La concentración elegida en el control reactivo (etanol) se definió en base a la mayor concentración utilizada en los extractos (5000 ppm). El porcentaje de mortalidad del control 
reactivo etanol fue de $1.1 \%$ (Figura 3 ), comprobando así que la mortalidad registrada en el bioensayo con los extractos etanólicos de hojas y semillas de $A$. muricata se debe principalmente a compuestos activos que influyen directamente en la muerte larval de A. aegypti.

El insecticida organofosforado (Abate ${ }^{\circledR}$ ) utilizado como control positivo alcanzó un porcentaje de mortalidad de $5.0 \%$. La dosis suministrada $(0.01562 \mathrm{~g} / 100 \mathrm{ml})$ se realizó en base a las recomendaciones de la OMS. El control negativo (agua) presentó el $0.2 \%$ de mortalidad durante el bioensayo, donde 100 individuos se expusieron a este control y solo 3 de ellos se registraron muertos. Estos valores de mortalidad posiblemente se deban a la susceptibilidad asociada a la densidad larval experimentada por los individuos durante el proceso de reproducción, tal como lo define (Francia A. y Maciá A., 2011).

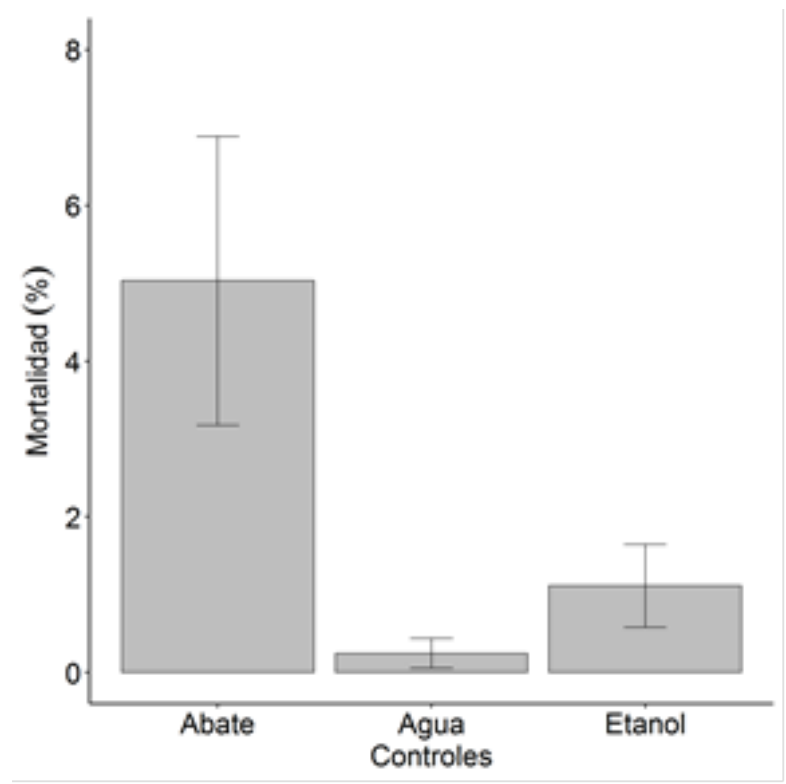

Figura 3. Mortalidad larval media ( \pm 1 error estándar) en función de los controles.

\section{Efecto de los parámetros físicos de los extractos etanólicos sobre la mortalidad de las larvas}

Se evaluó la temperatura y $\mathrm{pH}$ de los extractos etanólicos con el fin de monitorear las condiciones de desarrollo y sobrevivencia de las larvas. La temperatura es uno de los factores del cual dependen las larvas de A. aegypti, su traspase a estadio pupal se ve interrumpida si se encuentran en una temperatura menor de $13^{\circ} \mathrm{C}$, estos organismos son resistentes a temperaturas entre $10 \mathrm{a} 45^{\circ} \mathrm{C}$, sin embargo, se han planteado rangos de temperaturas óptimos para el desarrollo larval y oscilan entre 25 a $29^{\circ} \mathrm{C}$ (Eiman et al., 2016).

Los valores medios obtenidos reflejan que los rangos de temperatura de los extractos oscilaron entre 20.5 a $27^{\circ} \mathrm{C}$ para las matrices hojas y semillas. Las lecturas de estos parámetros 
realizadas en intervalos de 8 horas durante la evaluación del bioensayo se comportaron de forma estable (Figura 4).

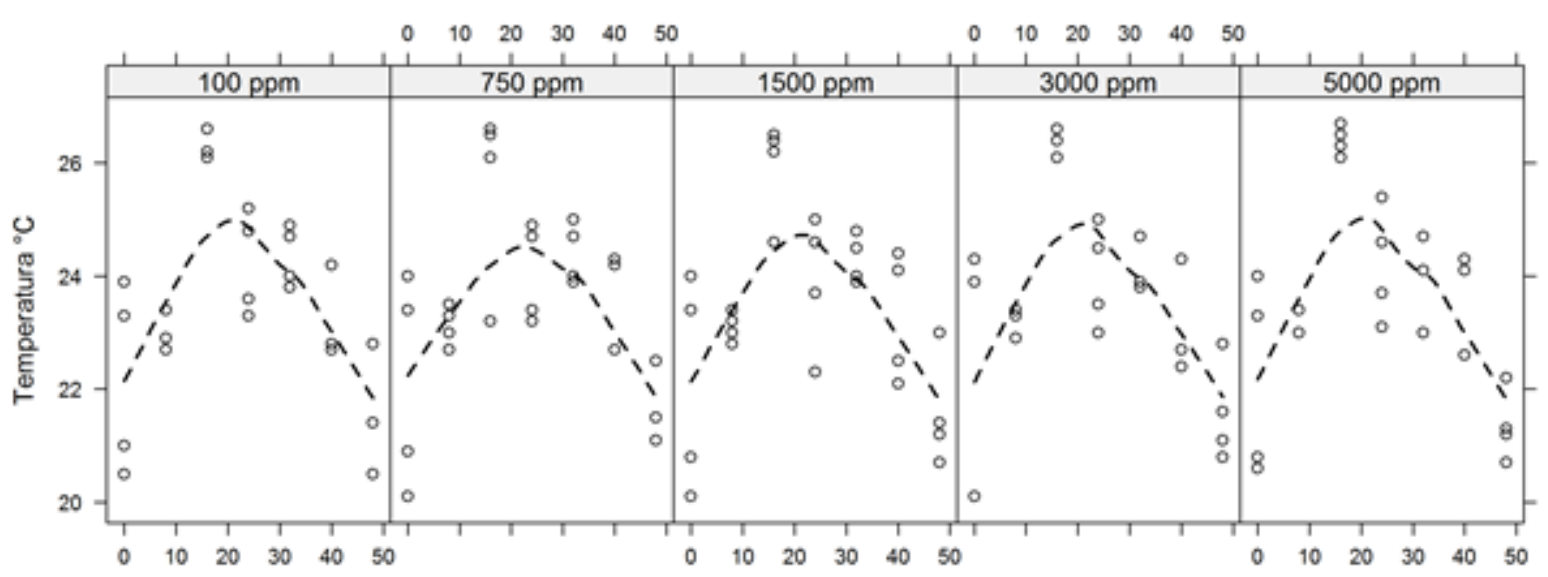

Figura 4. Temperatura del medio en cada concentración de A. muricata durante el bioensayo. La línea punteada representa una regresión ponderada localmente (LOESS).

El pH influye en el crecimiento larval. Díaz et al. (2015) describe que el nivel de tolerancia a los rangos de acidez va en dependencia del estadio larval. El pH ácido de 3.5 influye de manera directa aumentando los porcentajes de mortalidad en larvas del estadio I, y se reduce en larvas del estadio IV. Los valores de $\mathrm{pH}$ durante el bioensayo presentaron un rango amplio (5 - 7), tanto para hojas como semillas, sin embargo, estos no afectan significativamente en la mortalidad larval considerando que el rango más bajo reportado fue 5.0.

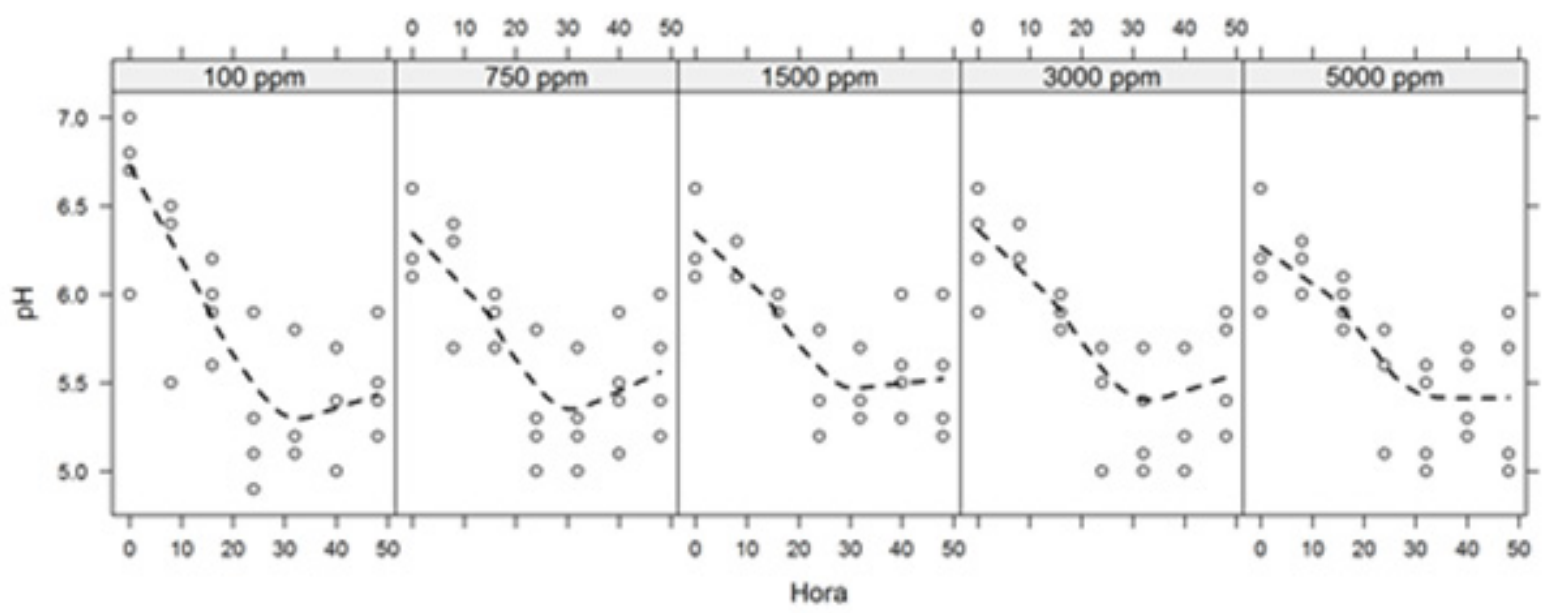

Figura 5. Valores de pH del medio en cada concentración de A. muricata durante la duración del bioensayo. La línea punteada representa una regresión ponderada localmente (LOESS).

\section{Efecto de las condiciones ambientales sobre la mortalidad de las larvas}

La temperatura y humedad relativa son otros factores que pueden incidir el desarrollo de A. aegypti en la fase acuática hasta su estado adulto. Diversas referencias internacionales 
describen rangos de temperaturas y humedad variables, desde 22 a $27^{\circ} \mathrm{C}$ y $75-80 \%$ (Quispe et al., 2014). Los valores de temperatura y humedad relativa reportados en el bioensayo presentan disminución y aumento de valores entre las áreas asignadas, esto se debe al periodo de lectura entre (día/noche), sin embargo, se encuentran dentro de los rangos reportados para el desarrollo de las larvas.
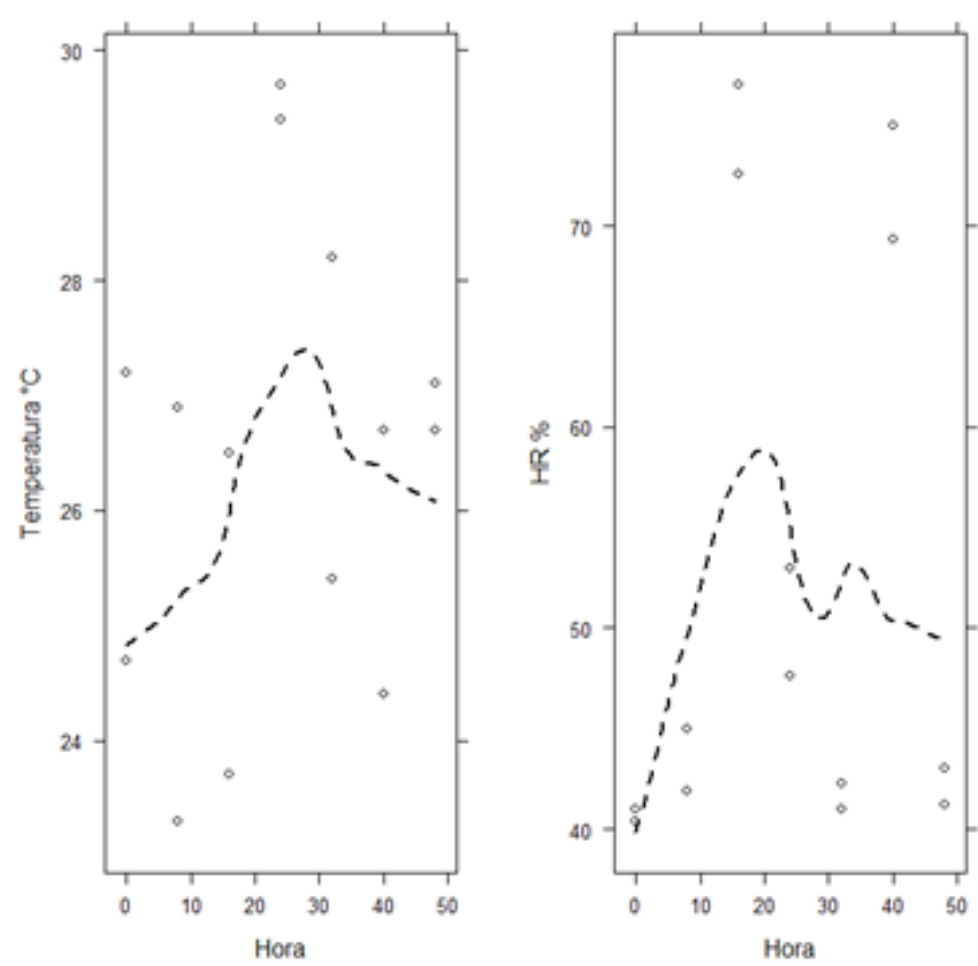

Figura 6. Temperatura y humedad relativa durante el bioensayo. La línea punteada representa una regresión ponderada localmente (LOESS).

\section{CONCLUSIONES}

Los resultados permitieron identificar que las diferencias en la composición proximal de las muestras están en dependencia no solo de la matriz (hoja o semilla) sino también de la procedencia de las mismas. Asimismo, se constata el efecto larvicida de los extractos de semilla y hoja de guanábana sobre el estadio larval III del Aedes aegypti, aunque su efectividad está en dependencia del método de extracción. Los resultados obtenidos para el control reactivo (etanol) reflejaron que la mortalidad registrada en el bioensayo se debe principalmente a compuestos activos presentes en los extractos. Adicionalmente, los parámetros evaluados en los medios y en el ambiente se encuentran dentro de los rangos sugeridos para bioensayos con A. aegypti, no interfiriendo con la mortalidad de las larvas. 


\section{AGRADECIMIENTOS}

Especial agradecimiento al Fondo para Proyectos de Investigación (FPI) de la Universidad Nacional Autónoma de Nicaragua, Managua (UNAN-Managua) por el financiamiento de los recursos requeridos para la realización de este proyecto. De igual manera se agradece a la MSc. Emperatriz Lugo y al Complejo Nacional de Salud Concepción Palacios por su invaluable aporte al facilitar los huevos de los especímenes para el desarrollo de esta investigación.

\section{REFERENCIAS BIBLIOGRÁFICAS}

Alfaro, J. (2017). Caracterización epidemiológica de la epidemia de dengue en el distrito seis de Managua Nicaragua 2015, Monografía Universidad Nacional Autónoma de Nicaragua. P. 86.

Castillo, J y Ramos J. (2016). Evaluación de la eficacia temefos $\left(\right.$ Abate $\left.^{\circledR}\right)$ en condiciones de laboratorio y su utilidad en el control de larvas de Aedes aegypti, vector del dengue y Chikungunya en el barrio Bóer. Monografía Universidad Nacional Autónoma de Nicaragua.

Cuello M, Jaramillo G, Canchingre E, Pérez J, Castro C, Cabrera O. (2017). Determinación de componentes nutricionales presentes en las hojas secas de Annona muricata L. (guanábana). Revista Cumbres. 2017; 3(1): 09-28.

Eiman M., Introini M., Ripoll C. (2016). "Directrices para la prevención y control de Aedes aegypti”. Ministerio de Salud de la Nación Av. 9 de Julio 1925 (C1073ABA), Cdad. Autónoma de Bs. As., República Argentina.

Fasakin A., Fehintola E., Obijole O., Oseni O. (2007). Compositional analyses of the seed of sour sop, Annona muricata L., as a potential animal feed supplement. Scientific research and essays 3(10):521523.

Francia, A. y Maciá, A. (2011). Efectos de la competencia larval en los mosquitos de contenedores artificiales, Aedes aegypti y Culex pipiens (Diptera: Culicidae) en condiciones semi-controladas. Revista de la Sociedad Entomológica Argentina, 70 (3-4), 305-315.

Quispe E., et al. (2014). Ciclo biológico y Tabla de Vida de Aedes aegypti, en laboratorio: Trujillo (Perú), 2014. Revista Científica de Estudiantes. REBIOLEST 2015; 1(3): e47.

R Core Team. (2019). R: A language and environment for statistical computing. R Foundation for Statistical Computing, Vienna, Austria. URL https://www.Rproject.org/.

Vit P., Santiago B. y Pérez Pérez E. M. (2014). Composición química y actividad antioxidante de pulpa, hoja y semilla de guanábana (Annona muricata L.). Interciencia, 39(5), 350-353. 